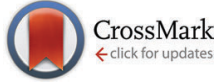

Cite this: Soft Matter, 2016, 12, 3654

DOI: $10.1039 / \mathrm{c} 6 \mathrm{sm} 90054 \mathrm{~b}$

www.rsc.org/softmatter

\title{
Correction: Revealing the dynamic heterogeneity of PMMA/PVDF blends: from microscopic dynamics to macroscopic properties
}

\author{
Bo Lu, ${ }^{a}$ Khalid Lamnawar, ${ }^{\text {b }}$ Abderrahim Maazouz ${ }^{\text {ac }}$ and Huagui Zhang ${ }^{\text {ad }}$
}

Correction for 'Revealing the dynamic heterogeneity of PMMA/PVDF blends: from microscopic dynamics to macroscopic properties' by Bo Lu et al., Soft Matter, 2016, DOI: 10.1039/c5sm02659h.

In the above manuscript, on page 4 the following text:

"Fortunately, this effect can be neglected since blends with higher PVDF fractions (e.g. 30/70 blend) and expected to be more thermally stable still show the TTS failure even in the frequency region up to the crossover frequency $\left(\omega_{c}\right)($ Fig. $2 d)$."

Should instead read:

"Fortunately, this effect can be neglected since blends with higher PVDF fractions (e.g. 30/70 blend) expected to be more thermally stable still show the TTS failure even in the frequency region up to the crossover frequency $\left(\omega_{\mathrm{c}}\right)$ (Fig. $\left.2 \mathrm{~d}\right) . "$

In the above manuscript, on page 4, the following text:

"Similar to the trend of $\eta_{0}$, the average relaxation times, the blends especially at intermediate compositions are shorter than those of the components and also exhibit a minimum for bends with $\phi_{\mathrm{PVDF}}=60-70 \%$, displaying a speed-up in relaxations."

Should instead read:

"Similar to the trend of $\eta_{0}$, the average relaxation times of blends especially at intermediate compositions are shorter than those of the components and also exhibit a minimum for bends with $\phi_{\mathrm{PVDF}}=60-70 \%$, displaying a speed-up in relaxations."

In the above manuscript, on page 5 , the following text:

"The observed curve up behaviour and a local minimum in viscosity, as well as the speed-up relaxations for blends with intermediate compositions, are assumed to be related to the reduced molecular entanglements, which will be discussed in the next section."

Should instead read:

"The observed concave up behaviour and a local minimum in viscosity, as well as the speed-up relaxations for blends with intermediate compositions, are assumed to be related to the reduced molecular entanglements, which will be discussed in the next section."

In the above manuscript, on page 5 , the following text:

"In particular, blends with intermediate compositions have the higher $M_{\mathrm{e}}$ and $M_{\mathrm{e} 12}$ and the maximum ones for $\phi_{\mathrm{PVDF}}=80 \%$, clearly suggest the reduced molecular entanglement; this is coincided with proceeding assumption, explaining the observed curve up behaviour and a local minimum in viscosity, and speed-up relaxations."

Should instead read:

"In particular, blends with intermediate compositions have the higher $M_{\mathrm{e}}$ and $M_{\mathrm{e} 12}$ and the maximum ones for $\phi_{\mathrm{PVDF}}=70 \%$, clearly suggesting the reduced molecular entanglement; this is coincided with proceeding assumption, explaining the observed concave up behaviour and a local minimum in viscosity, and speed-up relaxations."

The Royal Society of Chemistry apologises for these errors and any consequent inconvenience to authors and readers.

\footnotetext{
${ }^{a}$ Université de Lyon, CNRS, UMR 5223, Ingénierie des Matériaux Polymères, INSA Lyon, F-69621, Villeurbanne, France. E-mail: abderrahim.maazouz@insa-lyon.fr

${ }^{b}$ Université de Lyon, CNRS, UMR 5259, LaMCoS, Laboratoire de Mécanique des Contacts et des Structures, INSA Lyon, F-69621, Villeurbanne, France.

E-mail: khalid.lamnawar@insa-lyon.fr

${ }^{c}$ Hassan II Academy of Science and Technology, Rabat, Morocco

${ }^{d}$ School of Chemical and Process Engineering, University of Leeds, LS2 9JT, Leeds, UK
} 